\title{
Order parameter of a three-dimensional Ising-like system in the simplest and higher non-Gaussian approximations
}

\author{
I.V. Pylyuk* \\ Institute for Condensed Matter Physics of the National Academy of Sciences of Ukraine,
} 1 Svientsitskii St., 79011 Lviv, Ukraine

Received February 24, 2013, in final form April 10, 2013

\begin{abstract}
The application of the collective variables method to the study of the behaviour of nonuniversal characteristics of the system in the critical region is illustrated by an example of the order parameter. Explicit expressions for the order parameter (the average spin moment) of a three-dimensional uniaxial magnet are obtained in approximations of quartic and sextic non-Gaussian fluctuation distributions (the $\rho^{4}$ and $\rho^{6}$ models, respectively), taking into account confluent corrections. Some distinctive features appearing in the process of calculating the order parameter on the basis of two successive non-Gaussian approximations are indicated. The dependence of the average spin moment of an Ising-like system on the temperature and microscopic parameters is studied.
\end{abstract}

Key words: three-dimensional Ising-like system, critical behaviour, non-Gaussian measure density, order parameter

PACS: $05.50 .+q, 64.60 . F-, 75.10 . H k$

\section{Introduction}

The author is pleased and honored to contribute to the festschrift dedicated to the 60th anniversary of Professor Mykhailo Kozlovskii. Mykhailo Kozlovskii is an excellent scientist in the field of theoretical physics, one of the founders of a new approach to the description of phase transitions in statistical physics. This approach is beneficially used in the present publication.

The research is devoted to the theory of phase transitions and critical phenomena, which are the subjects of wide-range studies (see, for example, [1-5]). Close to a continuous phase transition, fluctuations on all length scales make contributions and render the perturbative approach problematic [6]. Therefore, one should resort to the non-perturbative methods [7] such as the exact and rigorous analytic solution methods (transfer matrix methods, combinatorial methods, Bethe-ansatz), conformal field theory analysis, non-perturbative renormalization-group (RG) analysis, numerical transfer-matrix calculations, Monte Carlo (MC) simulations. The exact solutions [8-10] and the conformal field analysis [11] are powerful tools to determine the critical exponents of two-dimensional models. However, these approaches are not helpful in most of the three-dimensional (3D) cases. The MC method can be used here. At present, we see a growing interest in the application of MC-based non-perturbative approaches and the development of powerful MC simulation tools for 3D systems [12, 13]. An advantage of the MC method is its capability of performing smart moves that can be tuned to each physical system or phenomenon. The MC method allows one to reach any desired accuracy. An alternative method, which has been successful in the study of phase transitions, particularly in two-dimensional systems, is that of numerical transfermatrix calculations combined with finite-size-scaling analysis [14]. This method is non-perturbative and does not require foreknowledge of special temperatures (such as the critical temperature) or of critical

\footnotetext{
*E-mail: piv@icmp.lviv.ua
} 
exponents. Two main formulations of the non-perturbative RG [the Wilson approach (also called WilsonPolchinski) and the "effective average action" approach] are discussed in resent publications [15-18]. The exact non-perturbative RG equations are known for various models on fractal lattices (see, for example, [19] and references therein). Moreover, a rigorous RG analysis has been made in four dimensions [20]. In most other cases, the non-perturbative RG equations cannot be solved exactly, and suitable truncations must be used, such as the derivative expansion [21-25] (limited to the small momentum behaviour) or its adaptation to arbitrary values of the momenta [26, 27].

In this paper, the mathematical description is performed within the method of collective variables (CV) [28, 29], which is similar to the Wilson non-perturbative RG approach (integration on fast modes and construction of an effective theory for slow modes) [30].

The object of investigation is a 3D Ising-like system with an exponentially decreasing interaction potential $\Phi\left(r_{\mathbf{i j}}\right)=A \exp \left(-r_{\mathbf{i j}} / b\right)$, where $A$ is a constant, $b$ is the radius of effective interaction, and $r_{\mathbf{i j}}$ is the distance between spins located at the sites $\mathbf{i}$ and $\mathbf{j}$ of a simple cubic lattice with period $c$ and $N$ sites (see, for example, [29, 31, 32]). The Ising model, which is simple and convenient for mathematical analysis, is widely used in the theory of phase transitions for the analysis of the properties of various magnetic and nonmagnetic systems (ferromagnets, antiferromagnets, ferroelectrics, binary mixtures, lattice model of liquids, etc.). The critical behaviour of the 3D Ising universality class was also discovered in systems with strong and electroweak interactions observed in high-energy physics.

The CV method allows one to calculate a partition function of the system and to obtain universal quantities (critical exponents) and nonuniversal characteristics (the phase-transition temperature $T_{\mathrm{c}}$ and thermodynamic functions near $T_{\mathrm{c}}$ ) by using a unified approach [28, 29, 32]. The available methods make it possible to calculate universal quantities to a quite high degree of accuracy (see, for example, [33-36]). An advantage of the CV method lies in the possibility to obtain and analyse the expressions for thermodynamic characteristics as functions of microscopic parameters of the system (i.e., the lattice constant and parameters of the interaction potential). The term collective variables is applied to a special class of variables specific to each individual physical system. The set of CV contains variables associated with the order parameters. For this reason, the phase space of CV is most natural in describing a phase transition. For magnetic systems, the $\mathrm{CV} \rho_{\mathbf{k}}$ are the variables associated with the modes of spin-moment density oscillations, while the order parameter is related to the variable $\rho_{0}$, in which the subscript "0" corresponds to the peak of the Fourier transform of the interaction potential. The CV method is based on the use of non-Gaussian measure densities [28, 29, 37]. A non-Gaussian density of measure at a zero external field is represented as an exponential function of the $\mathrm{CV}$, the argument of which contains, along with the quadratic term, higher even powers of the variable with the corresponding coupling constants. The simplest non-Gaussian measure density is the quartic one (the $\rho^{4}$ model) with the second and the fourth powers of the variable in the exponent. It is followed by the higher sextic measure density (the $\rho^{6}$ model) whose exponent includes the sixth power of the variable in addition to the second and the fourth powers, etc. The starting point of the problem statement in the CV method is the Hamiltonian of the system. After passing to the CV set, the Jacobian of the transition from the spin variables to the CV is calculated to obtain a partition function functional of the Ginzburg-Landau-Wilson type. The partition function of the spin system is integrated over the layers of the CV phase space. The main feature is the integration of short-wave spin-density oscillation modes, which is generally done without using perturbation theory. For this purpose, we divide the phase space of the $\mathrm{CV} \rho_{\mathbf{k}}$ into layers with the division parameter $s$. In each $n$th layer (corresponding to the region of wave vectors $B_{n+1}<k \leqslant B_{n}, B_{n+1}=B_{n} / s, s>1$ ), the Fourier transform of the potential is replaced by its average value (the arithmetic mean in the given case). To simplify the presentation, we assume that the correction for the potential averaging is zero, although it can be taken into account if necessary [28, 29]. The inclusion of this correction leads to a nonzero value of the critical exponent $\eta$ characterizing the behaviour of the pair-correlation function at the critical temperature $T_{\mathrm{c}}[29,38,39]$. As a result of step-by-step calculation of partition function, the number of integration variables in the expression for this quantity decreases gradually. The partition function is represented as a product of partial partition functions $Q_{n}$ of separate layers and the integral of the "smoothed" effective density of measure $\mathbb{W}_{2 m}^{(n+1)}(\rho)$ :

$$
Z=2^{N} 2^{\left(N_{n+1}-1\right) / 2} Q_{0} Q_{1} \cdots Q_{n}\left[Q\left(P_{n}\right)\right]^{N_{n+1}} \int \mathscr{W}_{2 m}^{(n+1)}(\rho)(\mathrm{d} \rho)^{N_{n+1}}
$$


Here, $N_{n+1}=N^{\prime} s^{-3(n+1)}, N^{\prime}=N s_{0}^{-3}, s_{0}=B / B^{\prime}, B^{\prime}=(b \sqrt{2})^{-1}$, and $B=\pi / c$ is the boundary of the Brillouin half-zone. The integrand

$$
\mathscr{W}_{2 m}^{(n+1)}(\rho)=\exp \left[-\frac{1}{2} \sum_{k \leqslant B_{n+1}} d_{n+1}(k) \rho_{\mathbf{k}} \rho_{-\mathbf{k}}-\sum_{l=2}^{m} \frac{a_{2 l}^{(n+1)}}{(2 l) ! N_{n+1}^{l-1}} \sum_{k_{1}, \ldots, k_{2 l} \leqslant B_{n+1}} \rho_{\mathbf{k}_{1}} \cdots \rho_{\mathbf{k}_{2 l}} \delta_{\mathbf{k}_{1}+\cdots+\mathbf{k}_{2 l}}\right]
$$

characterizes the corresponding non-Gaussian measure density of the $(n+1)$ th block structure (the $\rho^{2 m}$ model). At $m=2$, we have the quartic density of measure or the $\rho^{4}$ model. The case of $m=3$ corresponds to the effective sextic measure density or the $\rho^{6}$ model. In the exponent of the expression (1.2), the coefficient of the quadratic term $d_{n+1}(k)=a_{2}^{(n+1)}-\beta \tilde{\Phi}(k)$ depends on the inverse thermodynamic temperature $\beta=1 /(k T)$ as well as on the Fourier transform of the potential $\tilde{\Phi}(k)=\tilde{\Phi}(0)\left(1-2 b^{2} k^{2}\right), \delta_{\mathbf{k}_{1}+\cdots+\mathbf{k}_{2 l}}$ is the Kronecker symbol, $B_{n+1}=B^{\prime} s^{-(n+1)}$. The remaining quantities in expressions (1.1) and (1.2) are defined in [28, 29].

The approximations of quartic and sextic distributions for modes of spin-moment density oscillations are used in this paper in studying the behaviour of the spontaneous order parameter, which exists in the system at temperatures below the critical value of $T_{\mathrm{c}}$ (the low-temperature region). The scheme of calculating the order parameter is described for the two above-mentioned non-Gaussian approximations. Some distinctions connected with these approximations are revealed.

\section{Order parameter of the system in the $\rho^{4}$ model approximation}

The average spin moment is the order parameter of the system investigated. It is associated with the presence of a nonvanishing value of the variable $\rho_{0}$ for $T<T_{\mathrm{c}}$, at which there is an extremum of the integrand of the expression for the long-wave part of the partition function

$$
Z_{\mu_{\tau}+1}=\mathrm{e}^{-\beta F_{\mu^{+1}}^{\prime}} \int \exp \left[\beta \sqrt{N} \rho_{0} h+\tilde{B} \rho_{0}^{2}-\frac{G}{N} \rho_{0}^{4}\right] \mathrm{d} \rho_{0} .
$$

The number of the layer $\mu_{\tau}$ in the CV space characterizes the point of exit of the system from the criticalregime region at $T<T_{\mathrm{c}}$ [29]. The quantity $h$ is determined by the value of the constant external magnetic field $\mathscr{H}$ introduced in our analysis ( $h=\mu_{\mathrm{B}} \mathscr{H}, \mu_{\mathrm{B}}$ being the Bohr magneton). The expression for $-\beta F_{\mu_{\tau}+1}^{\prime}$ corresponding to the contribution to the free energy of the system from $\mathrm{CV} \rho_{\mathbf{k}}$ with the values of wave vectors $k \rightarrow 0$ (but not equal to zero) as well as the temperature-independent coefficients of the expressions

$$
\begin{aligned}
\tilde{B} & =\tilde{B}^{(0)}|\tau|^{2 v} \beta \tilde{\Phi}(0)\left[1+\tilde{B}^{(1)}|\tau|^{\Delta}+\tilde{B}^{(2)}|\tau|^{2 \Delta}-\tilde{B}^{(3)}|\tau|\right] \\
G & =G^{(0)}|\tau|^{v}[\beta \tilde{\Phi}(0)]^{2}\left[1+G^{(1)}|\tau|^{\Delta}+G^{(2)}|\tau|^{2 \Delta}-G^{(3)}|\tau|\right]
\end{aligned}
$$

are given in [29, 40]. Here, $v$ and $\Delta$ are the critical exponent of the correlation length and the exponent of the temperature confluent correction (i.e., the correction to scaling), respectively. The reduced temperature is defined by $\tau=\left(T-T_{\mathrm{c}}\right) / T_{\mathrm{c}}$. Performing in (2.1) the substitution of the variable

$$
\rho_{0}=\sqrt{N} \rho,
$$

we obtain

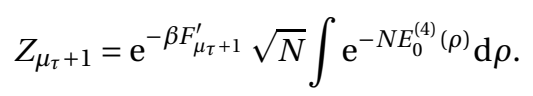

Owing to the factor $N$ in the exponent in (2.4), the integrand has a sharp maximum at the point $\bar{\rho}$ corresponding to the equilibrium value of the order parameter $\langle\sigma\rangle$. In the $\rho^{4}$ model approximation, the expression

$$
E_{0}^{(4)}(\rho)=G \rho^{4}-\tilde{B} \rho^{2}-\beta h \rho
$$

defines the fraction of free energy associated with the order parameter. It corresponds to a microscopic analogue of the Landau free energy. In contrast to the Landau theory, the temperature dependence of coefficients in (2.5) is nonanalytic [see (2.2)]. 
Thus, the evaluation of the order parameter $\langle\sigma\rangle$ is reduced to determining the extremum point $\bar{\rho}$ of the expression (2.5). In the case $h=0$, we find the following form for $\langle\sigma\rangle$ within the framework of the $\rho^{4}$ model:

$$
\langle\sigma\rangle=\sqrt{\frac{\tilde{B}}{2 G}}=\langle\sigma\rangle^{(0)}|\tau|^{v / 2}\left(1+\langle\sigma\rangle^{(1)}|\tau|^{\Delta}+\langle\sigma\rangle^{(2)}|\tau|^{2 \Delta}-\langle\sigma\rangle^{(3)}|\tau|\right) .
$$

All the coefficients in (2.6) are functions of microscopic parameters of the system, i.e., the effective radius of the potential $b$, the Fourier transform of the potential $\tilde{\Phi}(0)$ for $k=0$ and the lattice constant $c$. The expressions for the quantities $\langle\sigma\rangle^{(l)}(l=0,1,2,3)$ are presented in [40]. The values of these quantities for the effective interaction radius $b=c$ are contained in table 1 .

Table 1. Coefficients in expressions for the average spin moment of the system in approximations of the $\rho^{4}$ and $\rho^{6}$ models for some values of the RG parameter $s$.

\begin{tabular}{|c|c|c|c|c|c|c|}
\hline \multirow{2}{*}{$s$} & \multicolumn{4}{|c|}{$\rho^{4}$ model } & \multicolumn{2}{c|}{$\rho^{6}$ model } \\
\cline { 2 - 7 } & $\langle\sigma\rangle^{(0)}$ & $\langle\sigma\rangle^{(1)}$ & $\langle\sigma\rangle^{(2)}$ & $\langle\sigma\rangle^{(3)}$ & $\langle\sigma\rangle^{(0)}$ & $\langle\sigma\rangle^{(1)}$ \\
\hline \hline 2 & 0.4899 & 1.0809 & 1.0453 & 0.5042 & 0.3747 & 0.7485 \\
2.5 & 0.4384 & 0.8049 & 0.8438 & 0.5041 & 0.3387 & 0.5574 \\
3 & 0.3947 & 0.6528 & 0.6764 & 0.5039 & 0.3107 & 0.4651 \\
\hline
\end{tabular}

For an infinitely weak external field $h$, the average spin moment is defined by the formula

$$
\langle\sigma\rangle \approx c_{v}^{-1 / 2}\langle\sigma\rangle^{(0)} s^{-\left(\mu_{\tau}+1\right) / 2}\left(1+\langle\sigma\rangle_{1}|\tau|^{\Delta}+\langle\sigma\rangle_{2}|\tau|^{2 \Delta}-\frac{1}{2}|\tau|\right)\left(1-\frac{t}{3 \sqrt{3}}\right),
$$

where $c_{v}=\left(c_{1 k} / f_{0}\right)^{v}$. The temperature-independent quantities $c_{1 k}$ and $f_{0}$ characterize one of the coefficients in the solutions of recurrence relations for the $\rho^{4}$ model and one of the fixed-point coordinates, respectively [29]. The quantities $\langle\sigma\rangle_{1}$ and $\langle\sigma\rangle_{2}$ appear in the coefficients $\langle\sigma\rangle^{(1)}$ and $\langle\sigma\rangle^{(2)}$ from (2.6). The expression [40]

$$
t=-t^{(0)}\left(1+t^{(1)}|\tau|^{\Delta}+t^{(2)}|\tau|^{2 \Delta}\right)
$$

allows us to write down the average spin moment in the form

$$
\begin{aligned}
\langle\sigma\rangle \approx & c_{v}^{-1 / 2}\langle\sigma\rangle^{(0)} s^{-\left(\mu_{\tau}+1\right) / 2}\left\{1+\frac{t^{(0)}}{3 \sqrt{3}}+\left[\langle\sigma\rangle_{1}+\frac{t^{(0)}\left(\langle\sigma\rangle_{1}+t^{(1)}\right)}{3 \sqrt{3}}\right]|\tau|^{\Delta}\right. \\
& \left.+\left[\langle\sigma\rangle_{2}+\frac{t^{(0)}\left(\langle\sigma\rangle_{1} t^{(1)}+\langle\sigma\rangle_{2}+t^{(2)}\right)}{3 \sqrt{3}}\right]|\tau|^{2 \Delta}-\frac{1}{2}\left[1+\frac{t^{(0)}}{3 \sqrt{3}}\right]|\tau|\right\} .
\end{aligned}
$$

For $h=0$, the quantity $t^{(0)} \sim h$ vanishes, and the relation (2.9) goes over into (2.6).

\section{Average spin moment of the system in the $\rho^{6}$ model approximation}

In the previous section, the explicit expression for the average spin moment of the system is obtained on the basis of the $\rho^{4}$ model, taking into account the first and second confluent corrections (which are determined by the terms proportional to $|\tau|^{\Delta}$ and $|\tau|^{2 \Delta}$, respectively). In the present section, the calculation of the order parameter of the system in the higher non-Gaussian approximation (the $\rho^{6}$ model) involves the first confluent correction. by

We shall proceed from the expression coinciding in form with (2.4), where the role of $E_{0}^{(4)}(\rho)$ is played

$$
E_{0}^{(6)}(\rho)=D \rho^{6}+G \rho^{4}-\tilde{B} \rho^{2}-\beta h \rho .
$$


For the quantities appearing in (3.1), we can write the following relations accurate to within $|\tau|^{\Delta_{1}}$ with known coefficients [29]:

$$
\begin{aligned}
\tilde{B} & =\tilde{B}^{(0)}|\tau|^{2 v} \beta \tilde{\Phi}(0)\left[1+\tilde{B}^{(1)}|\tau|^{\Delta_{1}}\right], \\
G & =G^{(0)}|\tau|^{v}[\beta \tilde{\Phi}(0)]^{2}\left[1+G^{(1)}|\tau|^{\Delta_{1}}\right], \\
D & =D^{(0)}[\beta \tilde{\Phi}(0)]^{3}\left[1+D^{(1)}|\tau|^{\Delta_{1}}\right] .
\end{aligned}
$$

Here, the exponent of the first confluent correction $\Delta_{1}=-\ln E_{2} / \ln E_{1}$ in the case of the $\rho^{6}$ model is defined by the eigenvalues ( $E_{1}$ and $E_{2}$ ) of the RG linear transformation matrix (like $\Delta$ for the $\rho^{4}$ model). In contrast to the expression (2.5) (the $\rho^{4}$ model), the formula (3.1) contains the additional term, in which the coefficient $D$ is not equal to zero at $\tau=0$. Consequently, the expression (3.1) is most natural in describing the limiting case of $T=T_{\mathrm{c}}$ than the relation (2.5).

The value of $\bar{\rho}$ corresponding to the average spin moment can be determined from the condition of the extremum $\partial E_{0}^{(6)}(\rho) / \partial \rho=0$ or

$$
6 D \bar{\rho}^{5}+4 G \bar{\rho}^{3}-2 \tilde{B} \bar{\rho}-\frac{h}{k T}=0 .
$$

It is difficult to obtain the general solution of equation (3.3). However, this equation is easy to solve in some limiting cases. Let us consider the first case of this kind when there is no external field. For $h=0$, we obtain the biquadratic equation

$$
6 D \bar{\rho}^{4}+4 G \bar{\rho}^{2}-2 \tilde{B}=0
$$

in which the substitution of the variable

$$
\bar{\rho}^{2}=y
$$

leads to the equation

$$
6 D y^{2}+4 G y-2 \tilde{B}=0 .
$$

Solving this equation and separating temperature explicitly, we arrive at the following formula for the average spin moment $\langle\sigma\rangle=\bar{\rho}=\sqrt{y}$ :

$$
\langle\sigma\rangle=\langle\sigma\rangle^{(0)}|\tau|^{v / 2}\left(1+\langle\sigma\rangle^{(1)}|\tau|^{\Delta_{1}}\right) .
$$

The expressions for the coefficients $\langle\sigma\rangle^{(0)}$ and $\langle\sigma\rangle^{(1)}$ are given in [29, 41]. By analogy with the $\rho^{4}$ model, these coefficients are not universal since they depend on microscopic parameters of the system. The values of $\langle\sigma\rangle^{(0)}$ and $\langle\sigma\rangle^{(1)}$ (the case of the $\rho^{6}$ model when $b=c$ ) are presented in table 1

Let us now consider the second limiting case, $T=T_{\mathrm{c}}$ or $\tau=0$, when the solution of equation (3.3) can be written in a simple analytic form. Then, $\tilde{B}=0, G=0$ and we obtain the equation

$$
6 D \bar{\rho}^{5}=\frac{h}{k T} .
$$

In more compact form, it reads

$$
\bar{\rho}=\bar{\rho}_{0} h^{1 / \delta},
$$

where the critical exponent $\delta$ takes on the value 5 . The critical amplitude $\bar{\rho}_{0}$ satisfies the following relation:

$$
\bar{\rho}_{0}=\left\{6 k T_{\mathrm{c}} s_{0}^{6} D_{1}^{(0)}\left[\beta_{\mathrm{c}} \tilde{\Phi}(0)\right]^{3}\right\}^{-1 / 5} .
$$

Here, $\beta_{\mathrm{c}}=1 /\left(k T_{\mathrm{c}}\right)$ is the inverse phase-transition temperature. The quantity $D_{1}^{(0)}$ appears in the expression for $D$ from (3.2) in the coefficient $D^{(0)}=s_{0}^{6} D_{1}^{(0)}$. The solution (3.9) is obtained naturally. It cannot be found in a like manner within the framework of the $\rho^{4}$ model (for more details, see the next section).

The explicit expressions (2.6) and (3.7) make it possible to study the dependence of the order parameter of the system in approximations of the $\rho^{4}$ and $\rho^{6}$ models on the temperature and microscopic parameters. The temperature-dependence curves for $\langle\sigma\rangle$ near $T_{\mathrm{c}}$ are shown in figures 1 and 2 for different values of $s$ and $b$, respectively. Figure 1 demonstrates the results for the effective interaction radius $b=c$. As is seen from this figure, the dependence of the average spin moment on the RG parameter $s$ for 


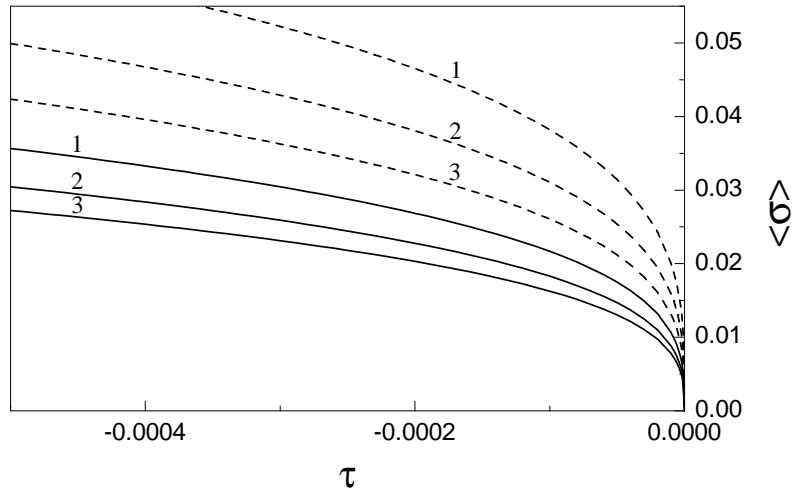

Figure 1. Temperature dependence of the average spin moment of the system for various values of the RG parameter $s$ within the framework of the $\rho^{4}$ model (dashed curves). For comparison, the average spin moment obtained in the $\rho^{6}$ model approximation without involving the confluent correction is also presented (solid curves). Curves 1,2, and 3 correspond to $s=2, s=2.5$, and $s=3$, respectively.

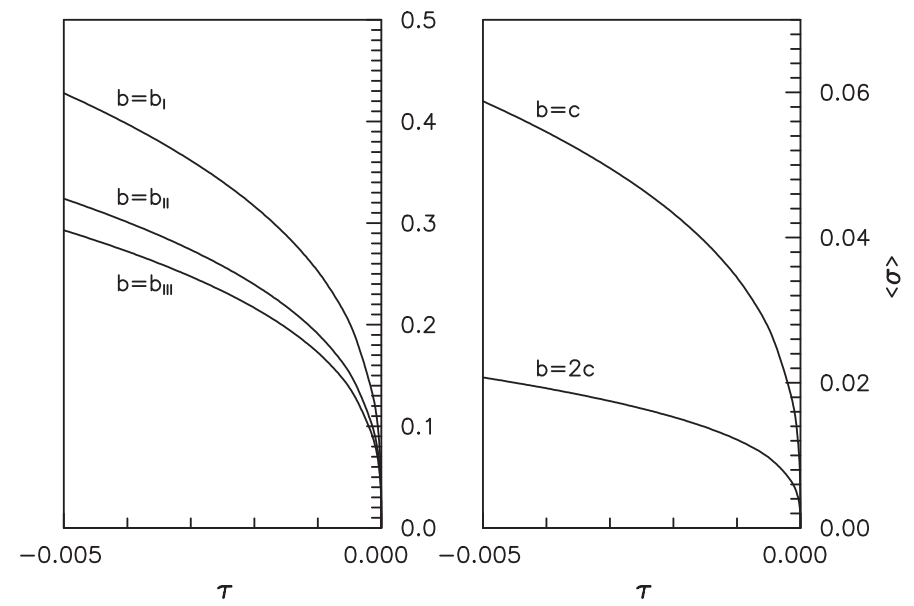

Figure 2. Dependence of the order parameter of the system on the reduced temperature in the $\rho^{6}$ model approximation for various values of the effective radius of the potential $b: b_{I}=c /(2 \sqrt{3}), b_{\mathrm{II}}=0.3379 c$, $b_{\text {III }}=0.3584 c, c$, and $2 c$.

the $\rho^{6}$ model is weaker than for the $\rho^{4}$ model. The $\rho^{6}$ model will be the key model in our further study. In figure 2 and below, the plots are obtained for the RG parameter $s=3$ with allowance for confluent corrections. At $s=3$, the numerical calculations are performed for $v=0.592, \Delta=0.475$ (the $\rho^{4}$ model) [42] and $v=0.640, \Delta_{1}=0.503$ (the $\rho^{6}$ model) [41]. The value of $b=b_{I}=c /(2 \sqrt{3})$ corresponds to the interaction between nearest neighbours, $b=b_{\text {II }}=0.3379 c$ corresponds to the interaction between the nearest and next-nearest neighbours, and $b=b_{\mathrm{III}}=0.3584 c$ corresponds to the nearest, next-nearest and third neighbours [29, 43]. At these values of $b$ and small values of the wave vectors $\mathbf{k}$, the parabolic approximation of the Fourier transform of an exponentially decreasing interaction potential corresponds to the analogous approximation of the Fourier transform for the interaction potentials of the above-mentioned neighbours. The evolution of the order parameter $\langle\sigma\rangle$ [see (3.7), the case of the $\rho^{6}$ model] for $\tau=-10^{-3}$ with an increasing ratio $b / c$ is demonstrated by the curve in figure 3 .

The plots in figures 2 and 3 show that the value of the order parameter decreases as the potential effective radius $b$ increases. This is the expected result since the condition $b \gg c$ corresponds to the transition to the model with long-range interaction, which demonstrates a classical critical behaviour. In the classical case, the order parameter $\langle\sigma\rangle_{\mathrm{cl}} \sim|\tau|^{\beta_{\mathrm{cl}}}$ is smaller than the nonclassical order parameter $\langle\sigma\rangle \sim|\tau|^{\beta}\left(\beta_{\mathrm{cl}}=0.5, \beta \approx 0.3,|\tau| \ll 1\right)$. The distribution for modes of spin-moment density oscillations at $b \gg c$ acquires the Gaussian form [44]. 


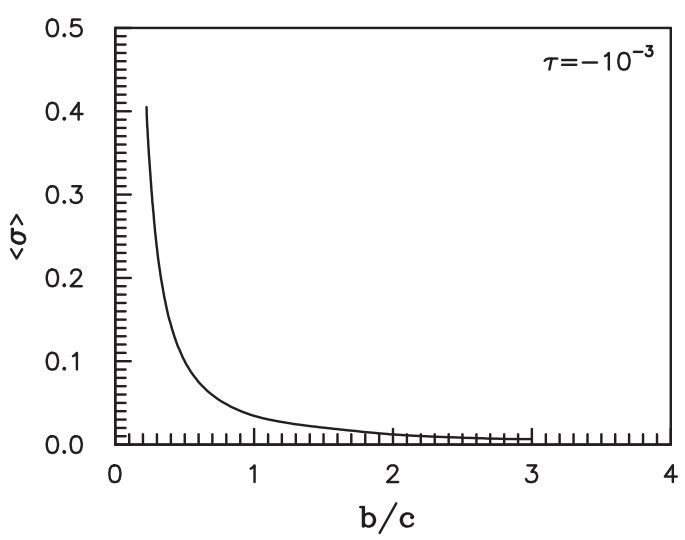

Figure 3. Behaviour of the average spin moment of the system for $\tau=-10^{-3}$ with an increasing ratio of the effective radius of an exponentially decreasing interaction potential to the simple cubic lattice constant (the $\rho^{6}$ model).

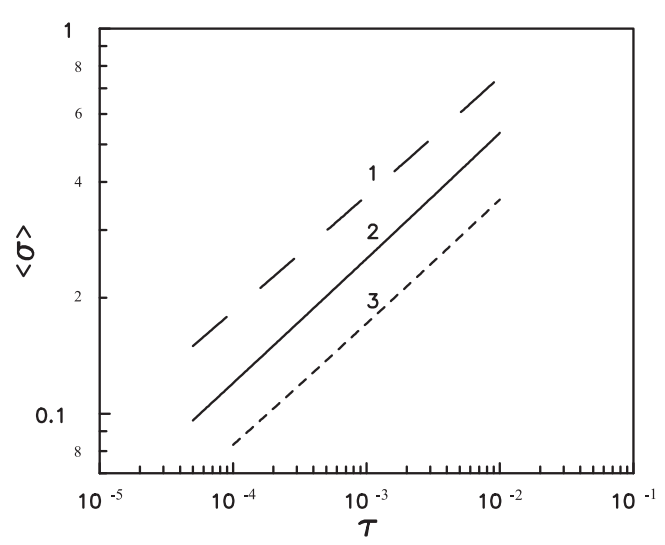

Figure 4. Order parameter of the 3D Ising model on a simple cubic lattice. Here, $\tau=\left|T-T_{\mathrm{C}}\right| / T_{\mathrm{C}}$. Straight line 1 corresponds to the $\rho^{4}$ model, line 2 corresponds to the $\rho^{6}$ model, and line 3 corresponds to the results obtained in [33].

The average spin moment calculated in approximations of the $\rho^{4}$ and $\rho^{6}$ models is in accord with the data obtained by other authors (see figure 4). The calculations were made for a simple cubic lattice in a zero external field with the interaction between nearest neighbours. In our calculations, we put $b=b_{\mathrm{I}}=c /(2 \sqrt{3})$. The straight lines 1 and 2 in figure 4 are parallel to line 3 . This is connected with the universality of critical exponents. As is clearly seen from figure 4 the plot for the $\rho^{6}$ model agrees more closely with the Liu and Fisher's results [33] than the estimates in the $\rho^{4}$ model approximation. Liu and Fisher in [33] carried out the numerical analysis of the leading critical amplitudes of susceptibility, correlation length, specific heat and spontaneous magnetization of 3D nearest-neighbour sc (simple cubic), bcc (body-centered cubic) and fcc (face-centered cubic) Ising models as well as universal relations between these amplitudes. Modern estimates of the critical temperature and exponents in [33] are used in conjunction with the biased inhomogeneous differential approximants to extrapolate the longest available series expansions to find the critical amplitudes.

\section{Discussion and conclusions}

The main interest of the expressions for thermodynamic functions, obtained within the CV method, is the direct connection of their coefficients with the fixed-point coordinates and the microscopic parameters of the initial Hamiltonian. This shows the capability of the CV method of being effective in describing both universal and nonuniversal characteristics of the system as functions of microscopic parameters. This capability is rather unusual with a RG approach since, for example, it is well known that the RG of perturbative field theory is only efficient in the close vicinity of the critical point and cannot account for a nonuniversal effect of a specific microscopic parameter of a specific system in a precise way. In that respect, the CV method is close to the Wilson non-perturbative RG where the pruning of the field theory (continuum limit) has not yet been performed. However, the CV method has an advantage over the Wilson approach, because it starts one step earlier (at the level of an explicit Hamiltonian) so that the microscopic parameters are directly accessible.

In the present paper, the order parameter (the average spin moment) of a 3D Ising-like system at $T<T_{\text {c }}$ is found using the simplest quartic (the $\rho^{4}$ model) and higher sextic (the $\rho^{6}$ model) non-Gaussian fluctuation distributions. The average spin moment of the system in these non-Gaussian approximations is determined as the solution of the corresponding equation. It should be noted that the solution (3.7) does not differ essentially from the similar one presented above for the case of the $\rho^{4}$ model [see [2.6)]. However, the solution (3.9) is obtained by means of a different route as compared to the $\rho^{4}$ model. In the latter case, one solves the cubic equation and excludes the reduced temperature $\tau$ [28]. For the $\rho^{6}$ 
model, the solution (3.9) is simply derived from equation (3.3) by nullifying $\tau$. This demonstrates that the mentioned solution in the limiting case of $\tau=0$ is obtained in more natural way for the $\rho^{6}$ model as compared to the $\rho^{4}$ one.

The expressions, presented in this paper in the simplest and higher non-Gaussian approximations, made it possible to study the behaviour of the average spin moment as a function of the temperature and microscopic parameters of the system. It should be emphasized that the dependence of the order parameter of the system on the RG parameter $s$ becomes weaker as the form of the non-Gaussian distribution for modes of spin-moment density oscillations becomes more complicated (transition from the $\rho^{4}$ model to the more complicated $\rho^{6}$ model). This is confirmed by a direct comparison of the curves describing the temperature dependence of the order parameter calculated for the $\rho^{4}$ and $\rho^{6}$ models at different values of the RG parameter $s$ (see figure 1). The rise in the effective radius of an exponentially decreasing interaction potential reduces the order parameter of the system (see figure2). The variation of the average spin moment with an increasing ratio of the potential effective radius to the simple cubic lattice constant is traced in the case of the $\rho^{6}$ model (see figure 3). Figures 2 and 3 are an illustration of the capability of the CV method of controlling the effects of microscopic parameters of the system.

The plots of the temperature dependence of the order parameter corresponding to the $\rho^{4}$ and $\rho^{6}$ models agree with the data of other authors (see figure 4). The employment of the $\rho^{6}$ model for the investigation of the phase transition by the $\mathrm{CV}$ method gives a more precise definition of the calculation results and provides the basis for the quantitative analysis of the critical behaviour of 3D Ising-like systems including the nonuniversal characteristics [29, 41]. For this more complicated $\rho^{6}$ model, the effect of microscopic parameters on the behaviour of the average spin moment in the critical region is revealed in the present study.

\section{References}

1. Baker G.A., Jr., Quantitative Theory of Critical Phenomena, Academic Press, San Diego, 1990.

2. Domb C., The Critical Point. A Historical Introduction to the Modern Theory of Critical Phenomena, Taylor \& Francis Ltd., London, 1996.

3. Kadanoff Leo P., Statistical Physics: Statics, Dynamics and Renormalization, World Scientific, Singapore, 2000.

4. Papon P., Leblond J., Meijer P.H.E., The Physics of Phase Transitions: Concepts and Applications, Springer, Berlin, 2006.

5. Nishimori H., Ortiz G., Elements of Phase Transitions and Critical Phenomena, Oxford University Press, Oxford, 2010.

6. Delamotte B., Canet L., Condens. Matter Phys., 2005, 8, No. 1(41), 163; doi 10.5488/CMP.8.1.163

7. Kaupužs J., Rimšāns J., Melnik R.V.N., Ukr. J. Phys., 2011, 56, No. 8, 845.

8. Onsager L., Phys. Rev., 1944, 65, No. 1, 117; doi 10.1103/PhysRev.65.117

9. McCoy B., Wu T.T., The Two-Dimensional Ising Model, Harvard University Press, Cambridge, 1973.

10. Baxter R.J., Exactly Solved Models in Statistical Mechanics, Academic Press, London, 1989.

11. Francesco P.D., Mathieu P., Sénéchal D., Conformal Field Theory, Springer, New York, 1997.

12. Preis T., Virnau P., Paul W., Schneider J.J., J. Comput. Phys., 2009, 228, No. 12, 4468; doi 10.1016/j.jcp.2009.03.018

13. Martinez E., Monasterio P.R., Marian J., J. Comput. Phys., 2011, 230, No. 4, 1359; doi 10.1016/j.jcp.2010.11.006

14. Richards H.L., Novotny M.A., Rikvold P.A., Phys. Rev. B, 1993, 48, No. 19, 14584; doi 10.1103/PhysRevB.48.14584

15. Bagnuls C., Bervillier C., Phys. Rep., 2001, 348, No. 1-2, 91; doi 10.1016/S0370-1573(00)00137-X

16. Berges J., Tetradis N., Wetterich C., Phys. Rep., 2002, 363, No. 4-6, 223; doi 10.1016/S0370-1573(01)00098-9

17. Delamotte B., In: Order, Disorder and Criticality. Advanced Problems of Phase Transition Theory, Vol. 2, ed. Holovatch Yu., World Scientific, Singapore, 2007, p. 1-78.

18. Caillol J.-M., Nucl. Phys. B, 2012, 855, No. 3, 854; doi 10.1016/j.nuclphysb.2011.10.026

19. Redinz J.A., Magalhães A.C.N. de, Phys. Rev. B, 1995, 51, No. 5, 2930; doi 10.1103/PhysRevB.51.2930.

20. Hara T., Tasaki H., J. Stat. Phys., 1987, 47, No. 1-2, 99; doi 10.1007/BF01009036

21. Aoki K.-I., Morikava K., Souma W., Sumi J.-I., Terao H., Progr. Theor. Phys., 1996, 95, No. 2, 409; doi 10.1143/PTP.95.409

22. Aoki K.-I., Morikava K., Souma W., Sumi J.-I., Terao H., Progr. Theor. Phys., 1998, 99, No. 3, 451; doi 10.1143/PTP.99.451

23. Morris T.R., Tighe J.F., J. High Energy Phys., 1999, 08, 007; doi 10.1088/1126-6708/1999/08/007

24. Morris T.R., Tighe J.F., Int. J. Mod. Phys. A, 2001, 16, No. 11, 2095; doi 10.1142/S0217751X01004761 
25. Canet L., Delamotte B., Mouhanna D., Vidal J., Phys. Rev. B, 2003, 68, No. 6, 064421; doi 10.1103/PhysRevB.68.064421

26. Blaizot J.-P., Méndez-Galain R., Wschebor N., Phys. Rev. E, 2006, 74, No. 5, 051116; doi 10.1103/PhysRevE.74.051116

27. Benitez F., Blaizot J.-P., Chaté H., Delamotte B., Méndez-Galain R., Wschebor N., Phys. Rev. E, 2012, 85, No. 2, 026707; doi 10.1103/PhysRevE.85.026707

28. Yukhnovskii I.R., Phase Transitions of the Second Order. Collective Variables Method, World Scientific, Singapore, 1987.

29. Yukhnovskii I.R., Kozlovskii M.P., Pylyuk I.V., Microscopic Theory of Phase Transitions in the Three-Dimensional Systems, Eurosvit, Lviv, 2001 (in Ukrainian).

30. Wilson K.G., Kogut J., Phys. Rep., 1974, 12, No. 2, 75; doi 10.1016/0370-1573(74)90023-4

31. Pylyuk I.V., Kozlovskii M.P., Condens. Matter Phys., 2001, 4, No. 1(25), 15; doi 10.5488/CMP.4.1.15

32. Yukhnovskii I.R., Pylyuk I.V., Kozlovskii M.P., J. Phys.: Condens. Matter, 2002, 14, No. 43, 10113; doi 10.1088/0953-8984/14/43/310

33. Liu A.J., Fisher M.E., Physica A, 1989, 156, No. 1, 35; doi 10.1016/0378-4371(89)90109-X

34. Landau D.P., Physica A, 1994, 205, No. 1-3, 41; doi 10.1016/0378-4371(94)90490-1

35. Guida R., Zinn-Justin J., J. Phys. A, 1998, 31, No. 40, 8103; doi 10.1088/0305-4470/31/40/006

36. Pelissetto A., Vicari E., Phys. Rep., 2002, 368, No. 6, 549; doi 10.1016/S0370-1573(02)00219-3

37. Yukhnovs'kii I.R., Riv. Nuovo Cimento, 1989, 12, No. 1, 1; doi 10.1007/BF02740597

38. Yukhnovskii I.R., Kozlovskii M.P., Pylyuk I.V., Ukr. J. Phys., 2012, 57, No. 1, 80 [Ukr. Fiz. Zh., 2012, 57, No. 1, 83 (in Ukrainian)].

39. Pylyuk I.V., Ulyak M.V., Condens. Matter Phys., 2012, 15, No. 4, 43006; doi 10.5488/CMP.15.43006.

40. Kozlovskii M.P., Pylyuk I.V., Yukhnovskii I.R., Theor. Math. Phys., 1991, 87, No. 3, 641; doi 10.1007/BF01017952 [Teor. Mat. Fiz., 1991, 87, No. 3, 434 (in Russian)].

41. Kozlovskii M.P., Pylyuk I.V., Dukhovii V.V., Condens. Matter Phys., 1997, 11, 17.

42. Kozlovskii M.P., Pylyuk I.V., Yukhnovskii I.R., Theor. Math. Phys., 1991, 87, No. 2, 540; doi 10.1007/BF01016127 [Teor. Mat. Fiz., 1991, 87, No. 2, 293 (in Russian)].

43. Kozlovskii M.P., Pylyuk I.V., Usatenko Z.E., Phys. Status Solidi B, 1996, 197, No. 2, 465; doi $10.1002 / p s s b .2221970221$

44. Pylyuk I.V., Low Temp. Phys., 1999, 25, No. 11, 877; doi 10.1063/1.593833 [Fiz. Nizk. Temp., 1999, 25, No. 11, 1170 (in Russian)].

\title{
Параметр порядку тривимірної ізингоподібної системи в найпростішому та вищому негаусових наближеннях
}

\begin{abstract}
I.В. Пилюк
Інститут фізики конденсованих систем НАН України, вул. Свєнціцького, 1, 79011 Львів, Україна

Застосування методу колективних змінних до вивчення поведінки неуніверсальних характеристик системи в критичній області проілюстровано на прикладі параметра порядку. Явні вирази для параметра порядку (середнього спінового моменту) тривимірного одновісного магнетика отримано в наближеннях четвірного та шестирного негаусових розподілів флуктуацій (моделі $\rho^{4}$ та $\rho^{6}$ відповідно) з врахуванням конфлуентних поправок. Вказано на деякі відмінні риси, які проявляються під час розрахунку параметра порядку на основі двох послідовних негаусових наближень. Досліджено залежність середнього спінового моменту ізингоподібної системи від температури та мікроскопічних параметрів.
\end{abstract}

Ключові слова: тривимірна ізингоподібна система, критична поведінка, негаусова густина міри, параметр порядку 


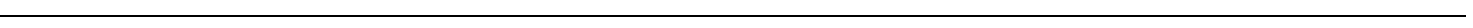

